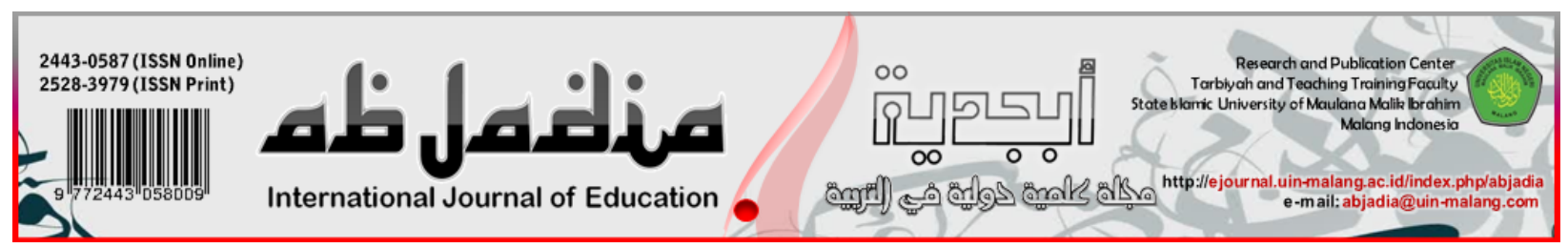

\title{
DEVELOPING A TEACHING PLAN FOR THE THIRD LISTENING SEMESTER USING THE COMMON EUROPEAN FRAMEWORK OF REFERENCE FOR LANGUAGES THROUGH BLENDED LEARNING
}

\author{
تطويرخطة تدريسية لفصل الاستماع الثالث باستخدام الإطار الأوروبي المرجعي الموحد للغات

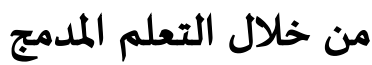

\author{
Riziq Fitra Pratama ${ }^{*}$, Muhammad Kamal bin Abdul Hakim², Syamsi Setiadi ${ }^{3}$ \\ 1,2,3 Universitas Negeri Jakarta
}

\begin{abstract}
Article History:
Received : 2021-01-10

Revised : 2021-04-24

Accepted : 2021-06-23

Published : 2021-06-30
\end{abstract}

\section{Keywords:}

Semester Learning Plan, Istima', CEFR, Blended Learning.

*Correspondence Address:

Bodayelshyrazy@gmail.com

\begin{abstract}
The scientific study was conducted in Arabic Language Study Program, Faculty of Languages and Arts, Jakarta State University, this study aims to produce development of RPS Istima' 3 (third listening) used by CEFR based on blended learning. The method used in this study is the development of ADDIE Model (Analysis, Design, Development, Implementation and Evaluation) developed by Dick and Carey. Which researcher limited the step to as follows: (1) Analysis; the need analysis of Arabic Language Study Program lecturers and students in Listening Course 3 CEFR-based Semester Lesson Plan through Blended Learning, (2) Design and Development; the researcher collected the listening course material of B-1 level based on CEFR which applied Blended Learning in teaching method and on previous Semester Lesson Plan, (3) Product's assessment was done by experts. The results show that the students' need analysis on CEFR-based Semester Lesson Plan of Listening Course 3 through Blended Learning scored at 2,46 in average which categorized 'very needed'. Furthermore, the expert review of the lesson plan gained 4 for its average which then defined as 'very good' category. Thus, CEFR-based Semester Lesson Plan of Listening Course 3 through Blended Learning is well worth using in Istima' 3 teaching and learning activities in Jakarta State University.
\end{abstract}

(C) مقلدمة

يتم تدريس اللغة العربية على نطاق واسع في المدارس الإسلامية في إندونيسيا التي تحتوي على محتوى مختلف. يمكن ملاحظة ذلك في منهج مدرسـة ثانوية لعام 19 ـr بناءً على قرار وزير الشؤون الدينية رقم

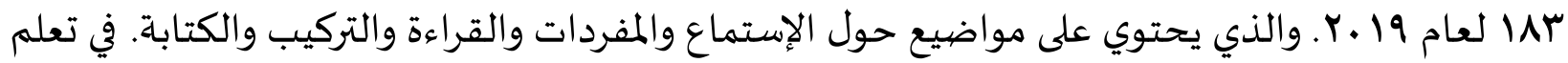
اللغة العببية، سيجد الطالب بعض المهارات اللغوية لإتقانها. تشمل المهارات اللغوية أربع مهارات، وهي مهارة الاستماع ومهارة الكلام ومهارة القراءة ومهارة الكتابة، وهذه المهارات الأربع تصبح جوانب مهمة في تعلم اللغة العربية، لأن هذه المهارات الأربعة لا تنفصل .موقف هذه المهارات الأربع مهمة في تحقيق المهارات اللغوية 
يتم تدريس المهارات اللغوية في بعض الجامعات التي تخصصت في اللغة العربية، مثل جامعة جاكرتا الحكومية. إحدى المهارات اللغوية التي يتم تعلمها في جامعة جاكرتا الحكومية هي مهارة الاستماع. ومهارة الاستماع لها ثلاثة مستويات، الاستماع الأوّل تسمى الاستماع الهامشي، الاستماع الثاني تسمى الاستماع المتواسط، الاستماع الثالث تسمى الاستماع الخييلي الناقد (Khalilullah, 2011, p. 39). وكل المستويات لها أهداف خاصية. ولذالك إن مهارة الاستماع هي مهارة مهمة، لأنه يتم تعلمها على مستويات مختلفة من التعليم، سواء في المدرسـة الثانوية أو الجامعة (Halimi, 2020).

مع مرور الوقت، يتغير تطوير عالم التعليم سريعا. أحد التغييرات المهمة التي حدثت في عالم التعليم في إندونيسيا هي تغيير المناهج الدراسية التي تتضمن أهداف تعليمية وجوانب تعليمية ومواد تعليمية. تحتوي المواد التعليمياة على خطة التدريس للفصل الدراسي (RPS). بالإشارة إلى المعايير الوطنية للتعليم العالي (SN-DIKTI)، كما هو منصوص في لائحة وزير بحوث تكنولوجيا والتعليم العالي رقم عء لعام 0 . ب المادة ا، يوضح أن خطة التدريس للفصل الدراسي هي مجموعة من الخطط والترتيبات لتحقيق مخرجات التعلم والمواد الدراسية والعمليات والتقييمات (Kemenristekdikti, 2015). لذلك، ركز الباحث بحثه على تطوير خطة تدريس للفصل الدراسي لمادة الاستماع الثالث في الفصل الرابع، التي أكمله المحاضر بناءً على أسـاس المناهج الدراسية الوطنية. الهدف من هذا التطوير هو أن الطلاب يمكنهم التواصل مع النّاطقين، وذلك اتِّال بالاستماع والكلام. ومن خلال إتقان مهارة الاستماع المناسبة، يمكن للطلاب فهم معاني الأساليب المسموعاة، وتفسير الجمل التي يتم استماعها، وتقديم تحليل للجمل المسموعاة، وفهم ما يستمعوناه تمامًا. تحتوي مادة الاستماع الثالث على إنجاز التعلم الموضيوعي (CPMK) الذي يهدف إلى فهم وتحليل الجمل العربية المسموعاة، ويستطيع تفسير الجمل المسموعة بشكل صحيح، وتحليل العلاقة بين الفقرات في الاستماع. ولكن بعد أن يأخذ الطلاب مادة الاستماع الثالث، لا يزال كثير منهم الذين لا يفهمون ويحللون جمل اللغة العربية بشكل كامل و لا يحللون العلاقة بين الفقرات في الاستماع و لا يفسرون الجمل التي يتم استماعها بشكل صحيح. من بعض الأسباب أن الطلاب لا يعرفون المفردات ولا يفهمون قواعد اللغة العربية. هذا يشير إلى ضعف الاهتمام بالطلاب في جامعة جاكرتا الحكومية. يحتاج الطلاب إلي إتقان المفردات وفهم قواعد اللغة العربية حتى يتمكنوا من فهم القراءة المسموعة من خلال الصيوت. لحل المشكلة، سيحاول الباحث تطوير خطة تدريس للفصول الدراس لمادة الاستماع الثالث باستخدام نظام يسمى الإطار الأوروبي المرجعي العام للغات (CEFR) وهو مجموعة من الوثائق المصهمة كمرجع لتعليم اللغة و التقييم. 
يستخدم الإطار الأوروبي المرجعي العام للغات للمساعدة في تطوير المهارات اللغوية مثل مهارة الاستماع ومهارة الكلام ومهارة القراءة ومهارة الكتابة بناءً على مستوى CEFR، وهو A1 و A2 يسمى المقياس الأول، B1 و B2 يسمى المقياس المتواسط، C1 و C2 يسمى المقياس التقدم. يستخدم الإطار الأوروبي المرجعي العام للغات على نطاق واسع في تحديد متطلبات إتقان اللغة. يعتمد على مركز الأنشطة اللغوية في نماذج وأنشطة اللغة (Alderson et al., 2006; Majlis Uwruba, 2008). لذلك يمكن أن يكون الإطار الأوروبي المرجعي العام للغات نموذجًا فعالًا جدًا لنظام يمكن تطبيقه في تعلم اللغة. في هذا العصر الحديث، تسهل التطورات التكنولوجية على المدرسين لتنفيذ التعلم بشكل غير مباشر أو ما يسهى بالتعلم الإلكتروني (E-Learning). وطريقة التعلم الإلكتروني مناسبة جدًا للطلاب الذين يجدوني

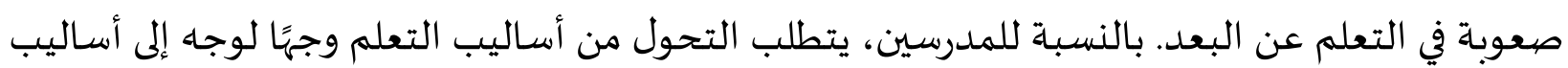
التعلم الإلكتروني الكثير من الجهد والمال. ولكن يمكن أن تبدأ بأساليب أحدث أو ما يسمى بالتعلم المدمج .(Badriyatul Muthoharoh, 2017)

والتعلم المدمج هو مزيج من طريقتين مختلفتين للتعلم في نفس الوقت. تجمع عملية التعلم المدمج في تطبيقها بين التعلم الإلكتروني والتعلم التقليدي من خلال طرق وجهاً لوجه. يمكن إجراء التعلم المختلط

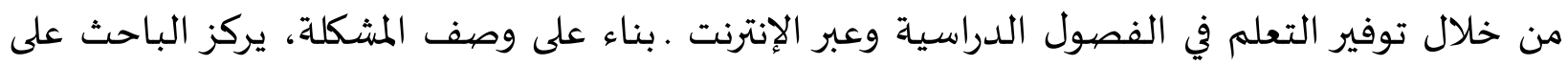
تطوير خطة التدريس للفصل الدراسيّ لمادة الاستماع الثالث في قسم تعليم اللغة العربية بجامعة جاكرتا الحكومية باستخدام الإطار الأوروبي المرجعي العام للغات من خلال التعليم المدمج.

\section{[ن]}

يستهدف هذا البحث للحصول على تطوير خطة التدريس لمادة الاستماع الثالث باستخدام الإطار الأوروبي المرجعي العام للغات (CEFR) من خلال التعليم المدمج (Blended Learning). لذلك استخدم هذا البحث طريقة البحث التطويري بالرمز ADDIE) Analyze, Develop, Design, Implement, and Evaluation.).

\begin{tabular}{|c|c|c|}
\hline البيان & الأنشطة & الخطوة \\
\hline تحليل احتياجات الطلاب على خطة التدريس & تحليل احتياجات & 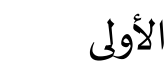 \\
\hline للفصل الدراسيّ لمادة الاستماع الثالث & الطلاب - اب & \\
\hline باستخدام الإطار الأوروبي المرجعي العام للغات & & \\
\hline من خلال التعليم المدمج. & & \\
\hline
\end{tabular}


تصميم خطة التدريس للفصل الدراسيّ لمادة

الاستماع باستخدام الإطار الأوروبي المرجعي العام للغات من خلال التعليم المدمج.

تقييم الخبراء على خطة التدريس للفصل الدراسيّ لمادة الاستماع الثالث باستخدام

الإطار الأوروبي المرجعي العام للغات من خلال

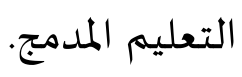

الثانية التديميم خطة

تقييم الخبراء

الثالثة

تتكون مجتمع هذا البحث عرشين طالبا في قسم تعليم اللغة العربية بجامعة جاكرتا الحكومية في الفصل الدرايس الخامس لمعرفة احتياجهم. واستخدم البحث أسلوب جمع البيانات بالاستبانة إلى عرشين طالبا على احتياجات خطة التدريس للفصل الدرايس لمادة الاستماع الثالث باستخدام الإطار الأوروبي المرجعي العام للغات من خلال التعليم المدمج، ثم قام بتقييم الخبريين عليه. وأخيرا، استخدم البحث التحليل الوصيفي لتقديم البيانات التي حصل عليها من نتائج الاستبانة. الجدول 2. تحليل المشاكل والصعوبات

\begin{tabular}{|c|c|c|}
\hline التقدير & النتيجة & الرقم \\
\hline سهل جـدا & $0,1-1,0$ & 1 \\
\hline سهل & $1,1-2,0$ & 2 \\
\hline صعب & $2,1-3,0$ & 3 \\
\hline صعب جـا & $3,1-4,0$ & 4 \\
\hline
\end{tabular}

\begin{tabular}{|c|c|c|}
\hline التقدير & النتيجة & الرقم \\
\hline غير محتاج & $0,1-1,0$ & 1 \\
\hline محتاج & $1,1-2,0$ & 2 \\
\hline محتاج جدا & $2,1-3,0$ & 3 \\
\hline
\end{tabular}




$$
\text { الجدول 4. المتوسط لمعايير التقييم من الخبراء }
$$

\begin{tabular}{|c|c|c|}
\hline التقدير & النتيجة & الرقم \\
\hline ضعيف & $0,1-1,0$ & 1 \\
\hline مقبول & $1,1-2,0$ & 2 \\
\hline جيد & $2,1-3,0$ & 3 \\
\hline جيد جدا & $3,1-4,0$ & 4 \\
\hline ممتاز & $4,1-5,0$ & 5 \\
\hline
\end{tabular}

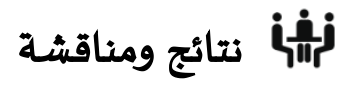
تحليل الإحتياجات

إن تعليم مهارة الاستماع عملية تحتاج إلل تعمق وإتقان، وليست هذه العملية نقل المعلومات من حيث النظرية بل أهم الأمور تريب مكثف ومتكين القواعد الأسـاسية وتناسب النظام الرئيس في الحصول علي كفاءة الطلبة ونجاحهم في تعلم هذه المهارة (Muhamad, 2018). أما تعليم الاستماع باستخدام باستخدام الإطار الأوروبي المرجعي العام للغات (CEFR) بمستوى B1 ، منها (1) فهم واستماع النص ابن بطوطة وأطول رحلة في التاريخ، (2) فهم واستماع النص مدينة غزة في التاريخ، (3) فهم واستماع النص ذكريات رمضيانية من الشام (4) فهم واستماع النص أعياد المسيحيين، (5) فهم واستماع النص من الأعياد في مصر، (6) فهم واستماع النص من التاريخ العربي الحديث، (7) فهم واستماع النص بدايات الصحافة العربية، (8) فهم واستماع النص الجزيرة الرأي والرأي الآخر، (9) فهم واستماع النص مهمة الجامعة، (10) فهم واستماع النص ندوة عن تاريخ الجزيرة العربية، (11) فهم واستماع النص الاحتلال الفرنسي للجزائر، فهم واستماع النص تاريخ الجامعات الاسلامية.

قام هذا البحث بتوزيع الاستبانة إلي الطلاب. وتوزيع الاستبانة لطلاب قسم تعليم اللغة العربية، جامعة جاكرتا الحكومية، بعدد عرشين طالبا في الفصل الدراسي الخامس الذين قد اشتركوا في مادة الاستماع الثالث. وحصل هذا البحث النتيجة أن الجواب لدى الطلاب بعد أن يتعلموا قدرتهم علي مهارات اللغة العربية، معظمههم يقولون جيد بعدد المتوسط 3,4. وبعد أن يتعلموا مادة الاستماع الثالث، معظمههم يقولون جيد بعدد المتوسط 3,3. 


\begin{tabular}{|c|c|c|c|}
\hline 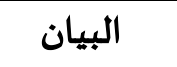 & النتيجة & 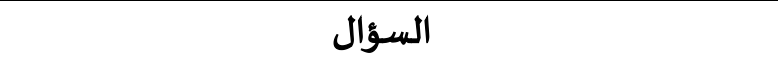 & 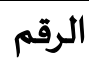 \\
\hline صعب & 2.6 & فهم واستماع النص ابن بطوطة وأطول رحلة في التاريخ & 1 \\
\hline صعب & 2.6 & فهم واستماع النص مدينة غزة في التاريخ & 2 \\
\hline صعب & 2.8 & فهم واستماع النص ذكريات رمضيانية من الشام & 3 \\
\hline صعب & 3 & فهم واستماع النص أعياد المسيحيين & 4 \\
\hline صعب & 2.9 & فهم واستماع النص من الأعياد في مصر & 5 \\
\hline صعب & 2.7 & فهم واستماع النص من التاريخ العربي الحديث & 6 \\
\hline صعب & 2.9 & فهم واستماع النص بدايات الصحافة العربية & 7 \\
\hline صبب جدا & $3 ، 2$ & فهم واستماع النص الجزيرة الرأي والرأي الآخر & 8 \\
\hline صعب & 2.6 & فهم واستماع النص مهمة الجامعة & 9 \\
\hline صعب جدا & 3.2 & فهم واستماع النص ندوة عن تاريخ الجزيرة العربية & 10 \\
\hline صعب جدا & 3.3 & فهم واستماع النص الاحتلال الفرنسي للجزائر & 11 \\
\hline صعب & 3 & فهم واستماع النص تاريخ الجامعات الاسلامية & 12 \\
\hline صعب & 2.89 & المعدل & \\
\hline
\end{tabular}

بناء على الجدول السابق عن تحليل المشكلات والصعوبات، وجد أن 3 عناصر من تحليل المشكلات والصعوبات صنفت على أنها صعبة جدًا، من بينها فهم واستماع النص الجزيرة الرأي والرأي الآخر بالنتيجة

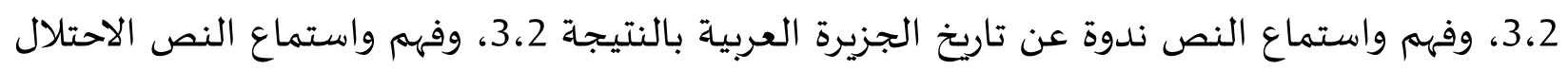
الفرنسي للجزائر بالنتيجة 3،3، بينما يتم تصنيف 9 عناصر من تحليل المشكلات والصعوبات صنفت على أنها صعب. والمعدل من الجدول السابق معظمهم يقولون صعب بالنتيجة 2،89. الجدول 7. تحليل الاحتياجات على خطة التدريس

\begin{tabular}{|c|c|c|c|}
\hline البيان & 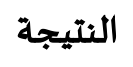 & 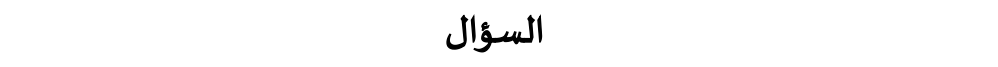 & 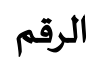 \\
\hline محتاج جدا & 2.7 & فهم وتقليد الخطاب العام المتعلق بالأدوات اليومية & 1 \\
\hline 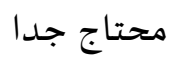 & 2.5 & فهم النقاط الرئيسية للمحادثة عن العمل في المكتب & 2 \\
\hline محتاج جدا & 2.4 & فهم النقاط الرئيسية للمحادثة عن الأنشطة في المطار & 3 \\
\hline 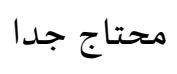 & 2.6 & فهم النقاط الرئيسية للنص عن الأنشطة الترفيهية & 4 \\
\hline 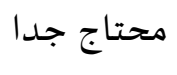 & 2.5 & فهم النقاط الرئيسية للنص عن الاهتمامات والهوايات & 5 \\
\hline محتاج جدا & 2.6 & فهم النقاط الرئيسية للفيلم عن تعلم اللغة العربية في المدرسة & 6 \\
\hline 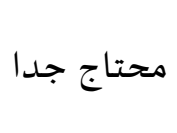 & 2.3 & 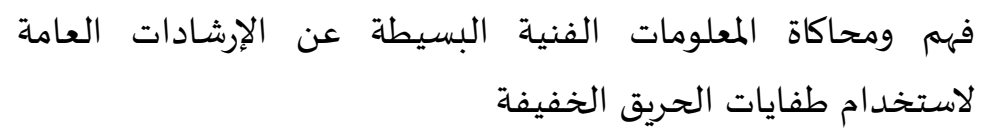 & 7 \\
\hline
\end{tabular}


Riziq Fitra Pratama, Muhammad Kamal bin Abdul Hakim, Syamsi Setiadi

\begin{tabular}{|c|c|c|c|}
\hline محتاج جدا & 2.4 & 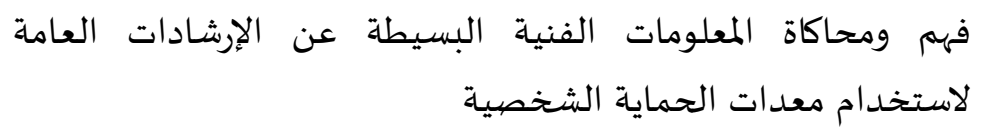 & 8 \\
\hline 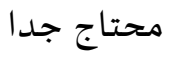 & 2.5 & فهم ومحاكاة المعلومات الفنية البسيطة عن إعلانات المنتجات التجارية & 9 \\
\hline محتاج جدا & 2.5 & فأثناء وباء كوفيد المعلومات التقنية البسيطة عن النداء للحفاظ على الصحة & 10 \\
\hline محتاج جدا & 2.5 & فوفهم وتحديد النقاط الرئيسية من الأخبار التلفزيونية عن تطوير لقاح & 11 \\
\hline محتاج جدا & 2.5 & فهم وتحديد النقاط الرئيسية من الأخبار التلفزيونية عن رياضية كرة & 12 \\
\hline 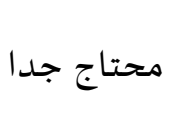 & 2.4 & فهم وتحديد النقاط الرئيسية من الأخبار التلفزيونية عن السياسة & 13 \\
\hline محتاج جدا & 2.46 & المعدل & \\
\hline
\end{tabular}

بناء على الجدول السابق عن تحليل الاحتياجات على خطة التدريس، وجد أن 13 عناصر من تحليل

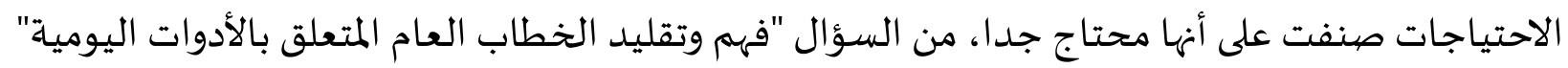
حصلت على النتيجة 7،2، وهي أكبر نتيجة في هذا التحليل. والمعدل من الجدول السابق بالنتيجة 46، 2 بالبيان محتاج جدا.

ومن البيان المذكور اكتشف الباحث أن الطلاب الذين يتعلمون الاستماع الثالث يحتاجون إلى خطة التدريس باستخدام الإطار الأوربى المرجعي العام للغات من خلال التعليم المدمج لأههم ناقصون في قدرتهم على مهارة الاستماع الثالث ولديهم المشكلات في ذلك.

\section{تصميم خطة التدريس}

إجابة عن المشكلات والصعوبات في تعليم مادة الإستماع الثالث السابقة والاحتياجات الطلاب على خطة التدريس، صمم الباحث خطة التدريس للفصل الدراسي لمادة الاستماع الثالث باستخدام الإطار الأوربى المرجعي العام للغات من خلال التعليم المدمج، في هذه المرحلة قام الباحث بجمع مهارات الاستماع في المستوى الثالث B-1 في الإطار الأوربى المرجعي العام للغات، التي لدهها الكفاءة في القدرة على التقاط وتقليد وفهم المعلومات الواقعية المباشرة حول الموضوعات اليومية العامة أو الأعمال ذات الصلة، وتحديد الرسائل العامة والتفاصيل المحددة. ثم يدخلها إلى خطة التدريس للفصل الدراسي لمادة الاستماع الثالث السابقة. 
الجدول 8. البيانات لدلالة على تصميم وتطوير خطة التدريس

\begin{tabular}{|c|c|}
\hline توضيع البيانات & \\
\hline 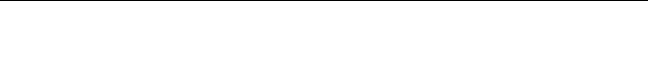 & الاستماع الثالث \\
\hline
\end{tabular}

$$
\text { الحكومية }
$$

تهدف هذه المادة إلى تزويد الطلاب بالقدرة على التقاط وتقليد

$$
4
$$

وفهم المعلومات الواقعية المباشرة حول الموضوعات اليومية

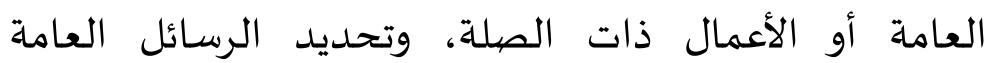

$$
\text { والتفاصيل المحددة. }
$$

تشمل هذه المادة: حوارات قياسية حول العمل المكتبي وأنشطة

المطار والأنشطة الترفيهية، ومقاطع فيديو للمادوات فياسية حول التعليمات البسيطة المكتي وانيطة

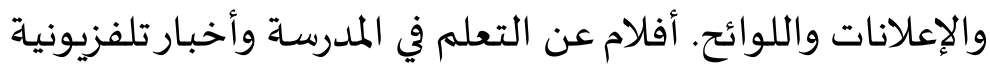

$$
\text { عن الأحداث الجارية. }
$$

5 ن 5

العمل في المكتب والمطار ووقت الفراغ والاهتمامات

$$
\text { والهوايات والأسرة. }
$$

2. قدرة على فهم والتعبير عن رسائل الأفلام باللغة العربية

$$
\text { عن التعلم في المدرسية. }
$$

3. قدرة على فهم ووصف مقاطع الفيديو باللغةرسة العربية

$$
\text { عن الأدوات اليومية. }
$$

4. قدرة على الاستماع وفهم المعلومات الهامة من المواد

$$
\text { السمعية والبصرية العربية. }
$$

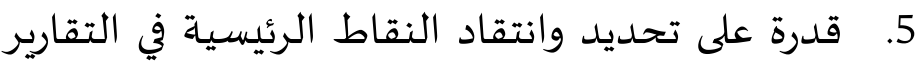

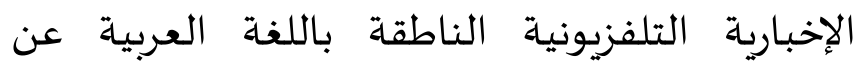

الأحداث الإخبارية الجارية مثل تطوير لقاح كوفيد 19،

$$
\begin{aligned}
& \text { والوضع الاجتماعي والسياسي في العالم. }
\end{aligned}
$$

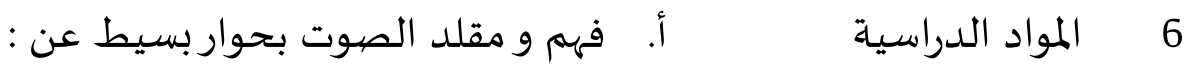

$$
\begin{aligned}
& \text { 1. العمل في المكتب } \\
& \text { 2. المحادثة في المطار }
\end{aligned}
$$




$$
\begin{aligned}
& \text { 3. الأنشطة في أوقات الفراغ } \\
& \text { 4. الاهتمامات والهوايات } \\
& \text { 5. 5. - 5. الأسـرة } \\
& \text { ب. فهم وكشف رسالة الفيلم عن : } \\
& \text { 1. تعلم اللغة العربية في المدرسة } \\
& \text { ج. فهم وشرح مقاطع فيديو عربية عن: } \\
& \text { 1. تعليمات بسيطة لاستخدام مطفأة الحريق } \\
& \text { 2. إعلان منتج تجاري } \\
& \text { 3. نداء للحفاظ على الصحة }
\end{aligned}
$$

د. تحديد وانتقاد النقاط الرئيسية في الأخبار التلفزيونية

$$
\text { 1. تطوير لقاح كوفيد } 19
$$

2. الظروف الاجتماعية والسياسية في العالم

يجري التعليم باستخدام المنهج التعليم النشط والتعليم المدمج.

يعد الطلاب للبحث والإيجاد والحصول عليها في سماع وفهم المعلومات الواقعية المباشرة، التعليم بشكل عام يستخدم

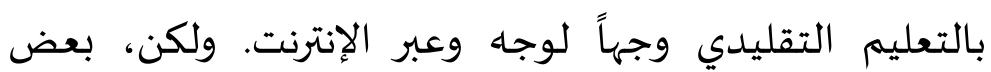
الواجبات المنظمة والمستقلة يجري بعبر الإنترنت. لذلك، يستخدم التعليم التعلم المدمج، وهو مزيج من التعليم التلقيني والتعليم الإلكتروني. يتم ضبط نسبة وجهاً لوجاه وعبر الإنترنت وفقًا لخصائص أهداف التعلم المراد تحقيقها. يناسب عدد التعليم

$$
\text { التلقيني والإلكتروني على الأهداف المطلوبة }
$$

شكل من أشكال عملية التعليم الطلاب المعرب عنها في الواجبات بحيث يتمكن الطلاب من تحقيق المهارات المتوقعة في كل مرحلة

$$
\text { من مراحل التعليم، وهي كالتالي : }
$$

1. مقدمة لتحديد الأصيوات العربية بشكل صحيح

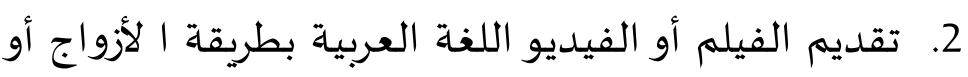

$$
\text { مجموعة }
$$

3. صياغة اسئلة وأجوبة الفيديو اللغة العربية وتلاوته.

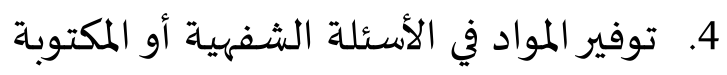

$$
9 \text { و و الإنترنت والكتب "العربية بينى يدك" والمعجم }
$$




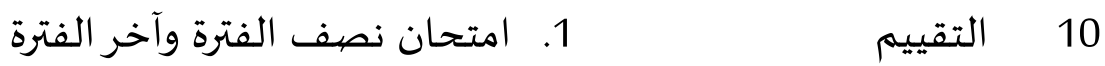

$$
\begin{aligned}
& \text { 2. 2 امتحان شفهي } \\
& \text { 3. الواجبات } \\
& \text { 4. }
\end{aligned}
$$

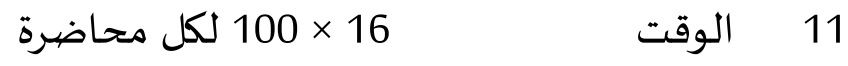

$$
\begin{aligned}
& 12
\end{aligned}
$$

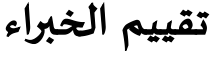

قام الباحث بتصحيح خطة التدريس للفصل الدراسي. هذا التحقيق من الدكتور محمد شريف الماجستير والدكتورة فتوى عريفة الماجستيرة. وهما المحاضران في قسم تعليم اللغة العربية. واستخدم الباحث الاستبانة بخمسـة أجوبة اختيارية (ممتاز، جيد جدا، مقبول، ناقص، راسب). والنتيجة المحصولة عليها

\begin{tabular}{|c|c|c|c|c|c|}
\hline البيان & المتوسط & الخبير الثاني & الخبير الأول & السؤال & 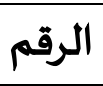 \\
\hline 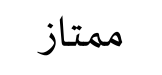 & 4.5 & 4 & 5 & صحيح الوصف & 1 \\
\hline جيد جدا & 3.5 & 3 & 4 & صحيح نتائج التعلم بالمادة & 2 \\
\hline جيد جدا & 3.5 & 3 & 4 & بالمادة صحيح نوع من نتائج التعلم & 3 \\
\hline ممتاز & 4.5 & 4 & 5 & مناسب بالإطار الأوربى & 4 \\
\hline جيد جدا & 4 & 3 & 5 & مناسب بالتعليم المدمج & 5 \\
\hline 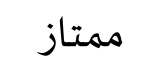 & 4.5 & 4 & 5 & مناسب بمعيار الوطني & 6 \\
\hline جيد جدا & 4 & 3 & 5 & بطالمادير نوع من نتائج التعلم & 7 \\
\hline جيد جدا & 3.5 & 3 & 4 & تطوير برنامج التعليم & 8 \\
\hline جيد جدا & 4 & 3 & 5 & تطوير طريقة التعليم & 9 \\
\hline ممتاز & 4.5 & 4 & 5 & مناسب نتائج التعلم بالمادة & 10 \\
\hline
\end{tabular}
الباحث منهما كما كتبها في الملحق. وجمع الإنتاجات كما في الجدول التالي : الجدول 9. تقييم استبانة من الخبيرين 


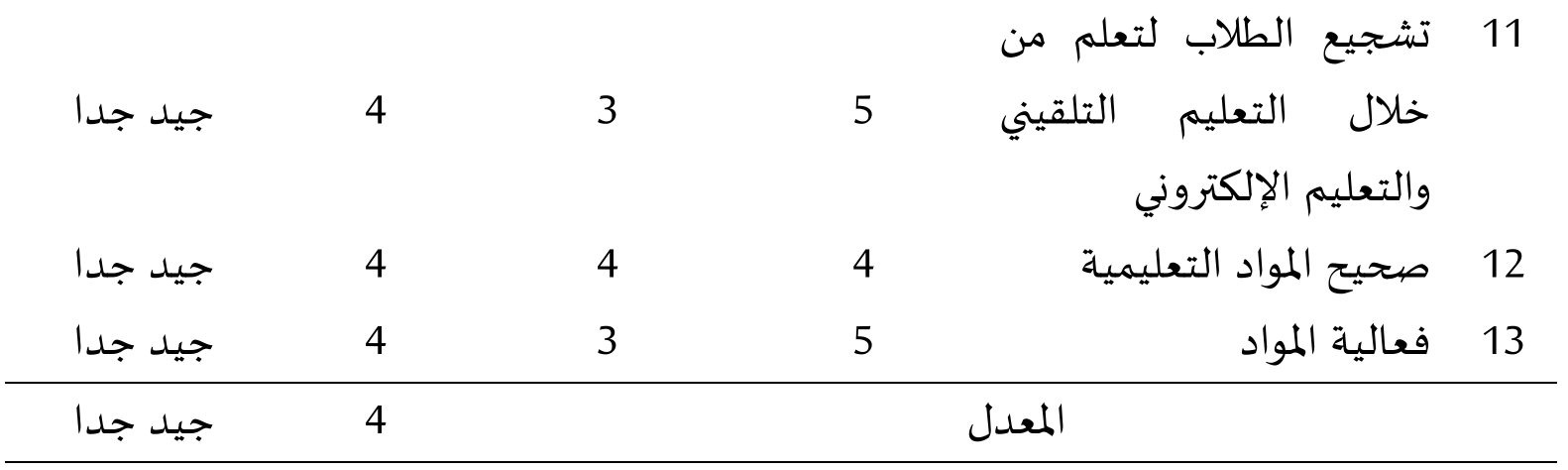

بالنسبة إلى الجدول السـابق أن التقييم من الخبيرين على خطة التدريس للفصل الدراسي لمادة الاستماع الثالث باستخدام الإطار الأوربى المرجعي العام للغات من خلال التعليم المدمج حصل على نتيجة المتوسط الإجمالي وبالنسبة إلى جدول المتوسط لمعايير التقييم من الخبراء، نتيجة تقييم من الخبيرين في مسافة3,1-4,0 حتّى تصل تقديرها "جيّد جدا". فلذلك، خطة التدريس لمادة الاستماع الثالث باستخدام الاطار الأروبي المرجعي العام للغات جيّد جدا بالنسبة إلى التقييم من الخبيرين.

\section{خاتمة 架}

يستهدف هذا البحث للحصول على تطوير خطة التدريس للفصل الدراسيّ لمادة الاستماع الثالث باستخدام الإطار الأوروبي المرجعي العام للغات من خلال التعليم المدمج. ونتائج البحث كما التالي: (1) تحليل الاحتياجات من الطلاب هو معظم الطلاب بعدد عشرون طالبا يجدون الصعوبة بالنتيجة 89، بالبيان الصعب ويحتاجون إلى خطة التدريس باستخدام الإطار الأوربى المرجعي العام للغات من خلال التعليم المدمج بالنتيجة 46،2 بالبيان محتاج جدا. (2) التقييم من الخبير الأول على خطة التدريس للفصل الدراسي لمادة الاستماع الثالث باستخدام الإطار الأوربى المرجعي العام للغات من خلال التعليم المدمج حصل على النتيجة 69،4 بالبيان ممتاز وصالح للاستعمال. والتقييم من الخبير الثاني على خطة التدريس للفصل الدراسي لمادة الاستماع الثالث باستخدام الإطار الأوربى المرجعي العام للغات من خلال التعليم المدمج حصل على النتيجة 38،3 بالبيان جيد جدا وصالج للاستعمال ولكن بالتصحيح ·

\section{مراجع}

Alderson, J. C., Figueras, N., Kuijper, H., Nold, G., Takala, S., \& Tardieu, C. (2006). Analysing Tests of Reading and Listening in Relation to the Common European Framework of Reference: The Experience of The Dutch CEFR Construct Project. Language Assessment Quarterly. https://doi.org/10.1207/s15434311laq0301_2 
Badriyatul Muthoharoh, N. (2017). Pengaruh Penggunaan Teknologi Pembelajaran Blended Learning Terhadap Hasil Belajar Menulis Teks Fungsional Pendek Bahasa Inggris. Deiksis.

Halimi, H. (2020). Preparing The Art Of Writing In The Style Of Arabic Literature For NonArabic Speakers / إعداد فن الكتابة بأسلوب الأدب العربي لغير الناطقين بالعربية. Ijaz Arabi Journal of Arabic Learning. https:// doi.org/10.18860/ijazarabi.v3i2.8782

Kemenristekdikti. (2015). Permenristedikti nomor 44 tahun 2015 tentang Standar Nasional Pendidikan Tinggi. Education.

Khalilullah, M. (2011). Strategi Pembelajaran Bahasa Arab Aktif (Kemahiran Istima' Dan Takallum). Jurnal Sosial Budaya, 1(2), 39.

Muhamad, F. (2018). Pembelajaran Maharah Istima'. Jurnal Komunikasi Dan Pendidikan Islam.

Taufik. (2016). Pembelajaran Bahasa Arab. In Okara.

Uwruba, M. (2016). Al'itar al-marja'i al-uwrubi al-'am li'lughat: Dirasah, tadris, taqyim. Cairo: Dar Ilyas al-'Asyriyah li-al-Tiba'ah wa-al-Nashr.

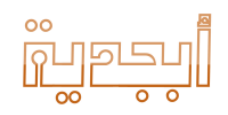

